\title{
The Nanofabrication and Application of Substrates for Surface-Enhanced Raman Scattering
}

\author{
Xian Zhang, ${ }^{1}$ Qin Zhou, ${ }^{1,2}$ Yu Huang, ${ }^{1}$ Zhengcao Li, ${ }^{1}$ and Zhengjun Zhang ${ }^{1}$ \\ ${ }^{1}$ Advanced Materials Laboratory, Department of Materials Science and Engineering, Tsinghua University, Beijing 100084, China \\ ${ }^{2}$ Institute of Nuclear and New Energy Technology, Tsinghua University, Beijing 100084, China \\ Correspondence should be addressed to Zhengjun Zhang, zjzhang@tsinghua.edu.cn
}

Received 8 September 2012; Revised 29 November 2012; Accepted 29 November 2012

Academic Editor: Rolf W. Berg

Copyright ( $) 2012$ Xian Zhang et al. This is an open access article distributed under the Creative Commons Attribution License, which permits unrestricted use, distribution, and reproduction in any medium, provided the original work is properly cited.

Surface-enhanced Raman scattering (SERS) was discovered in 1974 and impacted Raman spectroscopy and surface science. Although SERS has not been developed to be an applicable detection tool so far, nanotechnology has promoted its development in recent decades. The traditional SERS substrates, such as silver electrode, metal island film, and silver colloid, cannot be applied because of their enhancement factor or stability, but newly developed substrates, such as electrochemical deposition surface, Ag porous film, and surface-confined colloids, have better sensitivity and stability. Surface enhanced Raman scattering is applied in other fields such as detection of chemical pollutant, biomolecules, DNA, bacteria, and so forth. In this paper, the development of nanofabrication and application of surface-enhanced Ramans scattering substrate are discussed.

\section{Introduction}

Surface enhanced Raman Scattering was discovered by Fleischmann et al. in 1974 as a phenomenon where the intensity of Raman spectrum from pyridine adsorbed on a silver electrode is extrodinarily enhanced $[1,2]$. Then this effect was verified in many kinds of metals such as Ag, Au, $\mathrm{Cu}, \mathrm{Li}, \mathrm{Na}, \mathrm{K}$, and so forth $[2,3]$, while high enhancement is just found in $\mathrm{Ag}, \mathrm{Au}$, and $\mathrm{Cu}$. But the phenomenon was not correctly recognized at that time, then Jeanmaire and Van Duyne reported in 1977 that the enhancement of intensity of Raman spectrum from pyridine on silver electrode is attributed to the enhancement of the scattering cross-section of pyridine [4]. Then the phenomenon was accepted as surface enhanced Raman scattering (SERS).

Cross-sections of Raman scattering are typically 14 orders of magnitude smaller than those of fluorescene. So the weak intensity of Raman spectrum, the limits from the intensity of excitation sources, and the sensitivity of detector hindered the application of Raman spectrum for many years. The discovery of surface enhanced Raman scattering made Raman scattering more applicable in a wide range of research fields, while the interest and attention focused on the mechanism of SERS promotes the development of the theory on enhanced optical scattering from metal surface [57].

But SERS has not been developed to be a powerful detection technique so far, which is attributed to three main reasons [8-11]. First, the phenomenon-surface enhanced Raman scattering is obvious in only silver, gold, and copper, so the research and application of SERS ares limited. Second, there are limited kinds of processes that can achieve SERS substrate with high sensitivity successfully, and the practical SERS active substrate has not been realized. Third, the mechanism of surface enhanced Raman scattering is not totally clear.

Among so many theories about SERS that are proposed to explain the experimental characteristics of surface enhanced Raman scattering, it is widely accepted that the SERS effect mainly contributed to electromagnetic (EM) enhancement and chemical (CM) enhancement [12-19]. The electromagnetic (EM) enhancement is caused by local surface plasmon resonannce (LSPR) and depends on the material, shape, and size of the nanoscale substrate. There is a resonant frequency corresponding to a specific substrate, and the local surface plasmon resonance (LSPR) of substrate is driven into collective oscillator and generates the largest electromagnetic field when the light is incident 
with the resonant frequency. Electromagnetic enhancement relies on the location of molecules confined within the electromagnetic field and contributes an enhancement of more than $10^{5}$. While, the chemical enhancement is caused by a resonance Raman-like process associated with charge transfer between the analyte molecule and the metal surface and always contributes an enhancement factor of 100 . The charge transfer state increase the possibility of Raman transition by providing a pathway for resonant excitation. So chemical enhancement is dependent on site and analyte and happens just when the molecule is adsorbed directly on the metal roughened surface. Surface enhanced Raman scattering is generated from the combination of electromagnetic enhancement and chemical enhancement. However, SERS involves the complicated interactions and coupling between molecule and metal surface. So the theoretical work about SERS still attracts so much attention from scholars in different fields.

The research about surface enhanced Raman scattering revived in 1977, with the reported progress that Kneipp, Nie, and their coworkers brought SERS into the level of single molecule detection [20-23]. It is found that most SERS active substrate is nonuniform [24-28]. For instance, it is realized in silver colloid that nanoparticle with different sizes generates Raman signal with different enhancement factors [20]. From theoretical perspective, the highly active spots in SERS substrate are called "hotspots". The estimated SERS enhancement at hotspots reaches up to 14 orders, much higher than the enhancement factor achieved before; the result pushed research on SERS into a new era.

\section{The Nanofabrication of SERS Substrate}

The SERS substrate is the most important factor that affects the enhancement in SERS experiment, so the fabrication of SERS substrate always attracts most attention. Surface enhanced Raman scattering depends on the excitation of local surface plasmon resonance (LSPR), so it is critical to control the factors affecting LSPR such as the size, shape, material, interparticle spacing, and dielectric environment of substrate to improve its sensitivity and reproducibility [29]. By the efforts from many researchers, various kinds of SERS substrates are invented.

2.1. The Electrochemical Fabrication Method. Oxidationreduction cycle electrode [1] is the first found SERS electrode with reproducible enhancement factor of $10^{6}$, and it is widely applied in research on catalytic reactions and other electrochemically active systems [30]. Because of its low enhancement factor among the main kinds of SERS substrates, oxidation-reduction cycle electrode was not thought to be a very promising SERS substrate. But recently new process make roughen electrode more attractive. Duan et al. reported a simple, effective, and inexpensive method to fabricate an ordered Au particle array with hierarchical surface roughness on an indium tin oxide substrate based on an ordered alumina through-pore template [31]. The alumina template is produced by solution dipping on colloidal monolayer through an electrochemical deposition strategy. The array consists of periodically arranged and isolated $\mathrm{Au}$ microparticles, which show nanoscaled surface roughness. Essentially, this hierarchically rough particle array exhibits strong surface enhanced Raman scattering effect using Rhodamine 6G (R6G) as probe molecules, associated with its surface geometry. Aqueous solution of $10^{-6} \mathrm{M}$ R6G can be detected by such substrate. Huang et al. fabricated a kind of SERS substrate named Ag-capped Au nanopillar arrays by electrochemical deposition strategy [32]. Arrays of Au nanopillars with tube-like structures in the root parts were electrodeposited into the nanochannels of a porous AAO templates. After the AAO template is removed, the Ag is sputtered to adjust the space of Au nanopillar's tip. The $\mathrm{Au}$ nanopillar sputtered by $\mathrm{Ag}$ is proved to have capability of detecting $10^{-6} \mathrm{M}$ R6G.

\subsection{The Fabrication Method by Electron Beam Deposition.} The LSPR wavelength of metal island field can be tuned by changing the film's thickness and confluence, but the enhancement factor of metal island film is lower than other SERS substrate. Glancing angle deposition (GLAD) [33, 34] is a promising method to improve the morphology and SERS property of metal film. Zhou et al. fabricated aligned, single crystalline Ag nanorods on planar Si subatrates by GLAD technique, with sample substrate cooled by liquid nitrogen in e-beam deposition system [35]. It is successful to detect aqueous solution of $1 \times 10^{-12} \mathrm{~mol} \cdot \mathrm{L}^{-1}$ Rhodamine $6 \mathrm{G}$ by the porous Ag film with nanorods, the morphology and SERS performance of $\mathrm{Ag}$ film with aligned nanorods is shown in Figure 1. Then it is found that bends between two Ag arms with different orientations has better SERS sensitivity as hotspots, so the Ag film with nanorods of zigzag structure is an ideal way to obtain better performance [36].

Zhou et al. deposited Ag, Al, Si, and Ti thin films with thickness $d=25 \mathrm{~nm}, 100 \mathrm{~nm}$, and $400 \mathrm{~nm}$, respectively, to achieve layers with different reflectivity. Then Ag nanorods was deposited on the thin film layer. The SERS intensity of the Ag nanorods grown on Ag thin film are higher than others, and the SERS intensity of the Ag nanorods on $\mathrm{Al}$ film is larger than that on Ti film, and the Ag nanorods on Si film show the smallest SERS intensity. It is inferred that the larger the underlayer reflectivity, the larger the SERS performance of substrate. So the predeposition of Ag layer under Ag nanorods can be an effective way to promote the SERS performance of Ag nanorods [37].

It is realized that most SERS-active substrates are nonuniform before, and it is of specific interest to research hotspots where some spots and sites exhibit extrodinary SERS sensitivity and improve SERS substrate from randomly rough surface to highly ordered nanostructures [38]. Zhang et al. made Ag nanorods in film grow into periodic patterns at a micro-nano scale, and it is shown that the Ag nanorods film with periodic patterns exhibits better SERS performance than Ag film with nanorods arranged randomly as before [39]. The Ag film with periodic patterns is fabricated by the help of electron beam lithography. Hexagonal lattices $(200 \mu \mathrm{m} \times 200 \mu \mathrm{m})$ of silicon patterns $(\sim 1 \mu \mathrm{m}$ in diameter $)$ 


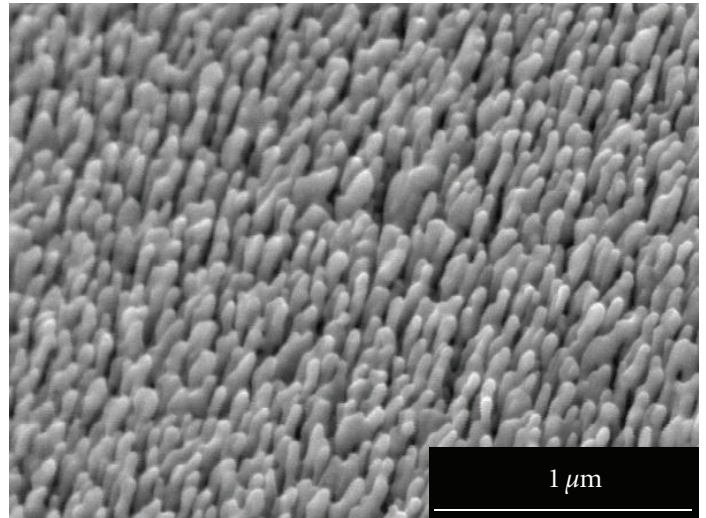

(a)

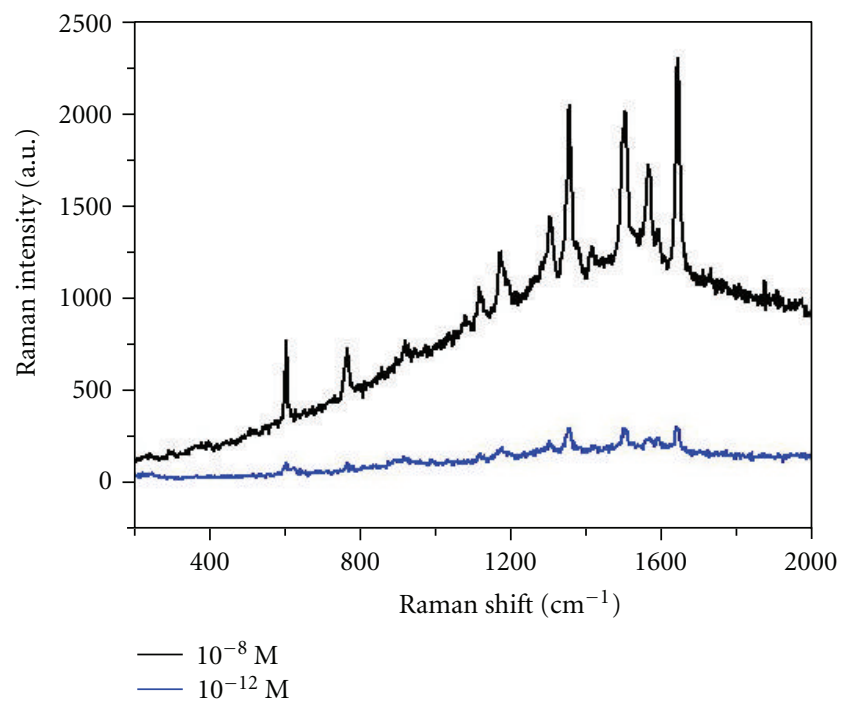

(b)

FIGURE 1: (a) The SEM image showing the morphology of porous Ag film on silicon substrate. (b) The Raman spectra of $10^{-8} \mathrm{M}$ and $10^{-12} \mathrm{M}$ Rhodamine 6G (R6G) in porous Ag film examined by Raman spectrometer.

were fabricated on the substrates by electron beam lithography, where the separation distance of the patterns was controlled to be 0 (closely-packed), $50 \mathrm{~nm}, 100 \mathrm{~nm}, 200 \mathrm{~nm}$, $300 \mathrm{~nm}, 400 \mathrm{~nm}, 500 \mathrm{~nm}$, and $600 \mathrm{~nm}$, respectively. Then Ag film with hexagonal patterns is deposited on silicon substrate with hexagonal patterns in a high vacuum ebeam deposition system, The morphology of Ag film with periodic patterns of different separation is shown in Figure 2. Characterized by Rhodamine 6G (R6G), the intensity of peak $612 \mathrm{~cm}^{-1}$ and $1508 \mathrm{~cm}^{-1}$ in Raman spectrum of R6G from Ag film with periodic patterns is shown in Figure 3. In comparison with signals from the planar area, it is noticed that when the patterns are closely packed $(0 \mathrm{~nm})$, or separated at $\sim 200 \mathrm{~nm}$, the signals are further enhanced by $\sim 20 \%$. When the diameter of periodic pattern in $\mathrm{Ag}$ film is $400 \mathrm{~nm}$ (as shown in Figure 4), different regulation of SERS performance is shown in Figure 5. In comparison with the unpatterned area, we notice that when the patterns are separated at $\sim 200 \mathrm{~nm}$, the signals are further enhanced by $\sim 5$ times compared with the unpatterned substrates. And the dependence behavior of the two peaks on the separation distance for pattern diameter of both $1 \mu \mathrm{m}$ and $400 \mathrm{~nm}$ is proved to be reproducible. So the SERS performance of Ag film with patterns' seperation of $200 \mathrm{~nm}$ is the best both among the Ag film with other separation, when the diameter of patterns is $1 \mu \mathrm{m}$ or $400 \mathrm{~nm}$. So it is demonstrated that the diameter and separation of patterns in $\mathrm{Ag}$ film both have effects on the SERS performance of Ag film, and the phenomenon suggests possibly a coupling effect at nanomicro scales on the localized electrical field. Furthermore, Chu et al. fabricated a gold disk array on a continuous gold film with silica spacer and demonstrated strong coupling between LSPs and surface plasmon polaritons (SPPs) [40]. It is predicted by simulations that the enhancement factor of each disk in array above a gold film is twice as large as that of a single disk above a gold film.

2.3. Other Fabrication Method. Colloidal nanoparticle substrate is easy to synthesize without sophisticated instrumentation and realizes single molecule detection initiatively. But colloidal nanoparticle substrate is unstable, so inmobilized colloidal nanoparticle is developed. Tong et al. synthesized self-assembly colloidal gold nanoparticles, and investigated the electromagnetic enhancement coupling effect appearing within the self-assembly nanoparticles [41].

Electron beam lithography substrates have been used to investigate the magnitude of the enhancement factor while the interparticle separation is varied, enhancement factors as large as $10^{8}$ have been achieved [42]. A soft technique known as nanosphere lithography has been used to fabricate SERS substrates to research atomic clusters, biomolecules, and redox couples in ultrahigh vacuum (UHV), electrochemically controlled, and ambient environments [43-45]. Van Duyne et al. used nanosphere lithography to fabricate surface confined Ag nanoparticles. Nanosphere lithography is an excellent approach to fabrication nanoparticle arrays with shape, size, and interparticle spacing precisely controlled. Meanwhile, metal film over nanosphere surfaces provides extremely SERS sensitivity for applications, and it is found that nanosphere lithography derived nanoparticles exhibit strong UV-visible extinction bands that are different from those of bulk metal. The LSPR spectra of nanosphere lithography derived nanoparticles can easily be tuned from the near-UV to the mid-IR by changing the size or shape of the nanoparticles [46]. Researchers continue to develop new SERS substrates to improve the sensitivity and reproducibility of its performance.

\section{The Application of SERS Substrate}

SERS has enormous potential for the detection of chemical and biological agents. The well resolved bands from Raman scattering allow the identification of composites, and low strength of signal from water is beneficial for the detection of biological agents [38]. 


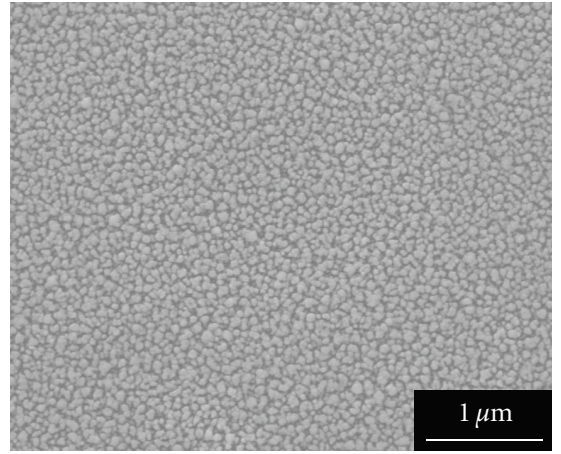

(a)

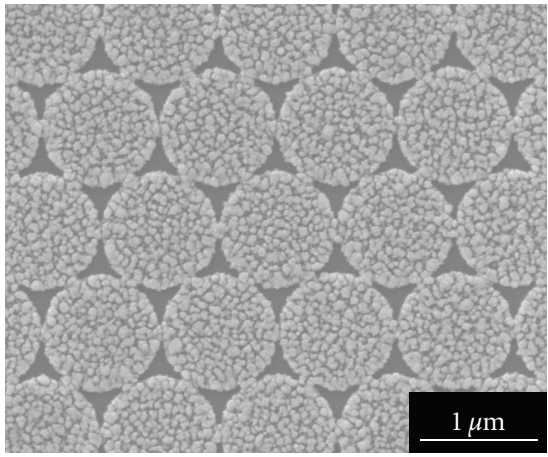

(b)

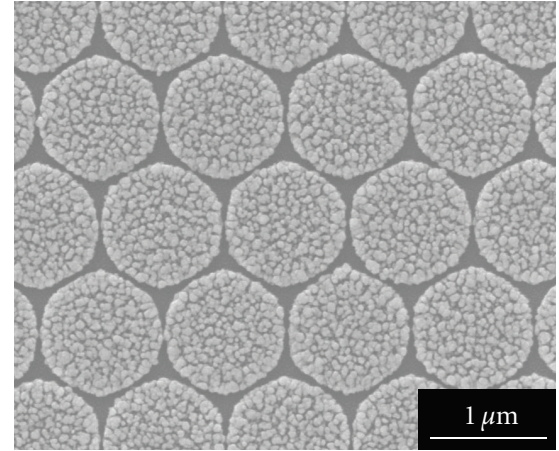

(c)

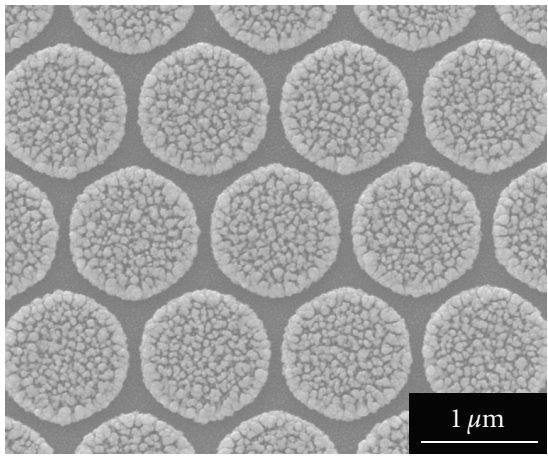

(d)

FIGURE 2: The SEM image of porous Ag film with different arrangement of nanorods (diameter of $1 \mu \mathrm{m}$ ). (a) Random arrangement; (b) periodical clusters packed closely; (c) periodical clusters with $50 \mathrm{~nm}$ separation; (d) periodical clusters with $200 \mathrm{~nm}$ separation.

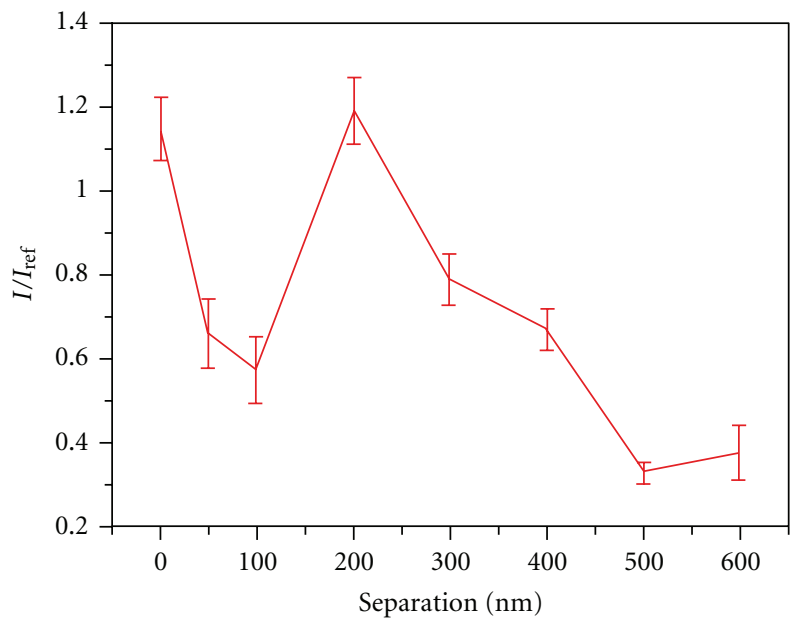

$-612 \mathrm{~cm}^{-1}$

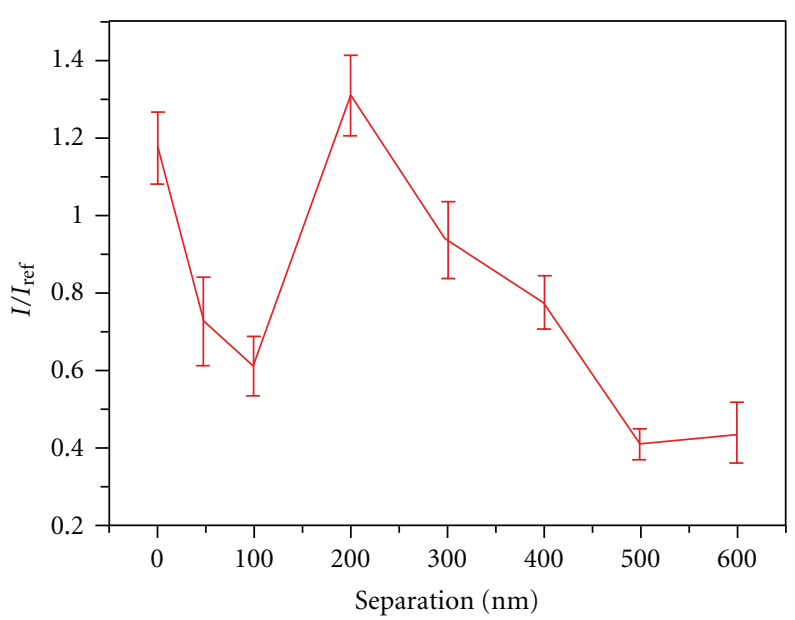

$-1508 \mathrm{~cm}^{-1}$

(a)

(b)

FIGURE 3: The intensity of the two characteristic peaks of $10^{-6} \mathrm{M}$ R6G on hexagonal lattices (with diameter of $1 \mu \mathrm{m}$ ) of Ag nanorods. (a) $612 \mathrm{~cm}^{-1}$ and (b) $1508 \mathrm{~cm}^{-1}$ that are normalized to the intensity obtained from the planar substrate.

It is a tendency that SERS substrate is made into sensor to facilitate the detection course. Van Duyne et al. has successfully made SERS substrate into a implantable glucose sensor, and the SERS substrate-silver surface is modified with a hydrophilic partition layer to realize the preconcentration of glucose within the range of electromagnetic field. Glucose was detected and quantified with the accuracy approaching the requirements for a biomedical device. And SERS has also 


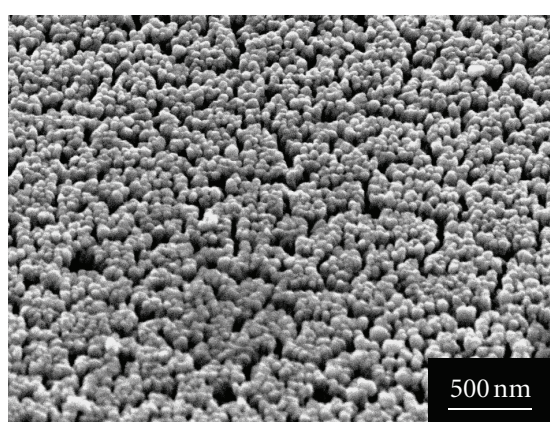

(a)

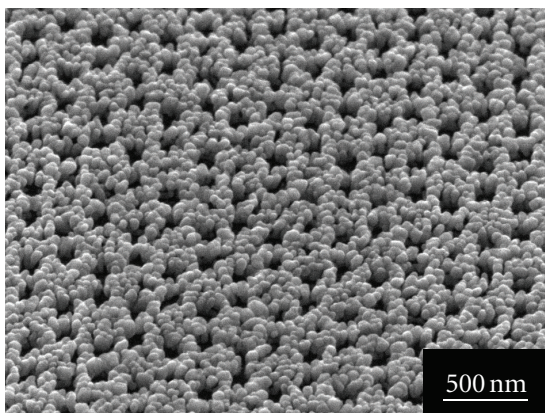

(b)

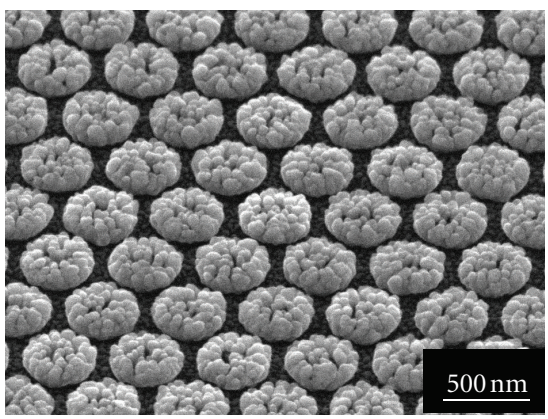

(d)

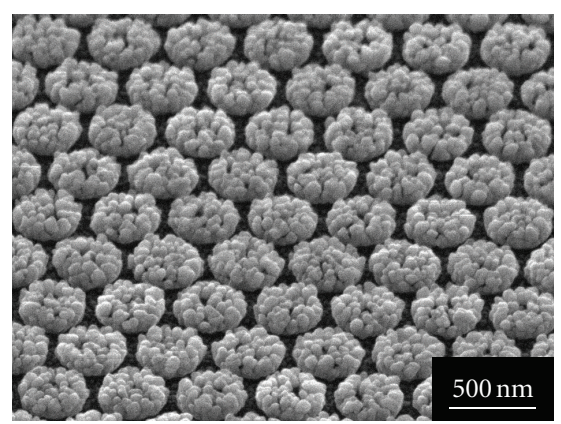

(c)

FIGURE 4: Oblique-view SEM images of vertically aligned Ag nanorods deposited on (a) unpatterned silicon; and on hexagonal lattices of silicon patterns that are separated by (b) $0 \mathrm{~nm}$ (closely-packed); (c) $50 \mathrm{~nm}$; (d) $100 \mathrm{~nm}$, with patterns diameter of $400 \mathrm{~nm}$.

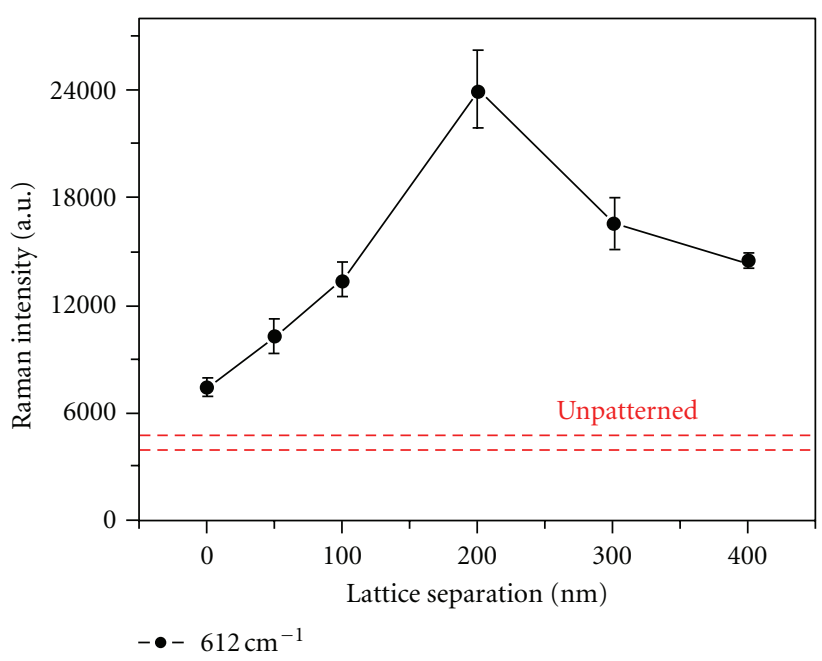

(a)

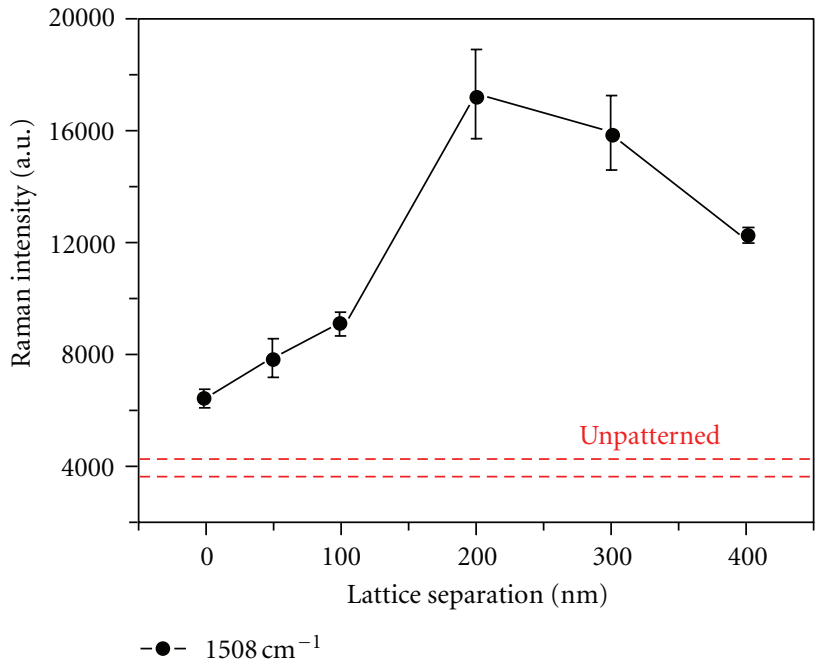

(b)

FIGURE 5: The intensity of the two characteristic peaks of $10^{-6} \mathrm{M}$ R6G on hexagonal lattices of Ag nanorods (with patterns diameter of $400 \mathrm{~nm}$ ): (a) $612 \mathrm{~cm}^{-1}$ and (b) $1508 \mathrm{~cm}^{-1}$.

been applied in military [47]. Silver nanowires were used to detect chemical warfare agents at a sensitivity of trace level [48].

Because of the excellent dielectric properties, polychlorinated biphenyls (PCBs) were used widely in transformers, heat transfer, capacitors, and so forth and had caused severe environmental pollution around the world [49]. It was found recently that PCBs are harmful to human body when the density exceeds the critical dose and PCBs can be accumulated through food chain from trace amount. While the most popular detection technique for PCBs is the combination of high-resolution gas chromatography and mass spectrometry, the method requires sophisticated devices, standard samples, complicated pretreatments of samples, proper experimental environment, and so forth. So new method that can detect PCBs at trace amount rapidly 
is necessary, and surface enhanced Raman scattering has potential to realize the goal.

Zhou et al. detected and distinguished the isomers of monochlorobiphenyls, tetrachlorobiphenyls, and 2,3,3',4,4' pentachlorinated biphenyls successfully [50-53]. To facilitate the distinguishing of polychlorobiphenyls, Zhou et al. simulated the vibration mode of polychlorobiphenyls by density functional theory, and the results are beneficial for distinguishing the agents after achieving the Raman spectrum.

Sometimes, the detection is carried out indirectly in biomolecules. For example, Cao et al. used ssDNA with a gold nanoparticle and SERS label to detect specific DNA molecule. If the probe is exposed to the target DNA, the hybridization of DNA occurred with SERS detection of the Raman label. The method detects the target DNA not by detection of DNA directly but by detection of Raman label's signal indirectly [54].

\section{Conclusion}

Recently, the development of fabrication and application of substrates for surface enhanced Raman scattering is driven by nanotechnology. More and more SERS substrates with high sensitivity and reproducibility are invented by electrochemical deposition, physical vapor deposition of metal film, metal nanoparticle colloids, and so forth and applied into various fields, such as detection of pollutants at trace level, surface analysis, biomolecule, bacteria detection, and so forth. With the development of SERS substrate, Raman spectrometer, and tip enhanced Raman scattering (as the combination of surface enhanced Raman scattering and atomic force microscopy), surface enhanced Raman scattering will be more and more applicable as a detection tool.

\section{Acknowledgments}

The authors are grateful to the financial support by the National Natural Science Foundation of China (50931002) and the National Basic Research Program of China (973 Program, 2007CB936601).

\section{References}

[1] M. Fleischmann, P. J. Hendra, and A. J. McQuillan, "Raman spectra of pyridine adsorbed at a silver electrode," Chemical Physics Letters, vol. 26, no. 2, pp. 163-166, 1974.

[2] M. Moskovits, "Surface-enhanced spectroscopy," Reviews of Modern Physics, vol. 57, no. 3, pp. 783-826, 1985.

[3] A. Kudelski, "Analytical applications of Raman spectroscopy," Talanta, vol. 76, no. 1, pp. 1-8, 2008.

[4] D. L. Jeanmaire and R. P. Van Duyne, "Surface Raman spectroelectrochemistry Part I. Heterocyclic, aromatic, and aliphatic amines adsorbed on the anodized silver electrode," Journal of Electroanalytical Chemistry, vol. 84, no. 1, pp. 1-20, 1977.

[5] R. K. Chang, "Surface enhanced Raman-scattering at electrodes-a status-report," Physical Chemistry Chemical Physics, vol. 91, no. 4, pp. 296-305, 1987.
[6] R. L. Garrell, "Surface-enhanced Raman spectroscopy," Analytical Chemistry, vol. 61, no. 6, pp. 401A-411A, 1989.

[7] M. Moskovits, "The dependence of the metal-molecule vibrational frequency on the mass of the adsorbate and its relevance to the role of adatoms in surface-enhanced Raman scattering," Chemical Physics Letters, vol. 98, no. 5, pp. 498-502, 1983.

[8] A. G. Brolo, M. Odziemkowski, and D. E. Irish, "An in situ Raman spectroscopic study of electrochemical processes in mercury-solution interphases," Journal of Raman Spectroscopy, vol. 29, no. 8, pp. 713-719, 1998.

[9] B. H. Loo, "Surface enhanced Raman scattering from pyridine adsorbed on cadmium," The Journal of Chemical Physics, vol. 75, no. 12, pp. 5955-5956, 1981.

[10] T. López-Ríos and Y. Gao, "Modification by pd adsorbates of the surface enhanced Raman scattering at Ag surfaces," Surface Science, vol. 205, no. 3, pp. 569-590, 1988.

[11] Y. Gao and T. Lopez-Rios, "Raman scattering of pyridine coadsorbed with Al on quenched Ag films: evidence of Raman enhancement in the pores," Surface Science, vol. 198, no. 3, pp. 509-523, 1988.

[12] M. Moskovits, "Surface-enhancedspectroscopy," Reviews of Modern Physics, vol. 57, no. 3, pp. 783-826, 1985.

[13] M. Moskovits, "Surface selection rules," Journal of Chemical Physics, vol. 77, no. 9, pp. 4408-4416, 1982.

[14] M. Moskovits, L. L. Tay, J. Yang, and T. Haslett, "SERS and the single molecule," in Topics in Applied Physics, pp. 215-226, Springer, Berlin, Germany, 2002.

[15] A. Otto, I. Mrozek, H. Grabhorn, and W. Akemann, "Surfaceenhanced Raman scattering," Journal of Physics, vol. 4, no. 5, pp. 1143-1212, 1992.

[16] M. Kerker, "Electromagnetic model for surface-enhanced Raman scattering (SERS) on metal colloids," Accounts of Chemical Research, vol. 17, no. 8, pp. 271-277, 1984.

[17] H. Metiu and P. Das, "The electromagnetic theory of surface enhanced spectroscopy," Annual Review of Physical Chemistry, vol. 35, pp. 507-536, 1984.

[18] G. C. Schatz, "Theoretical studies of surface enhanced Raman scattering," Accounts of Chemical Research, vol. 17, no. 10, pp. 370-376, 1984.

[19] J. R. Lombardi, R. L. Birke, T. H. Lu, and J. Xu, "Chargetransfer theory of surface enhanced Raman spectroscopy: Herzberg-Teller contributions," Journal of Chemical Physics, vol. 84, no. 8, pp. 4174-4180, 1986.

[20] S. Nie and S. R. Emory, "Probing single molecules and single nanoparticles by surface-enhanced Raman scattering," Science, vol. 275, no. 5303, pp. 1102-1106, 1997.

[21] K. Kneipp, Y. Wang, H. Kneipp et al., "Single molecule detection using surface-enhanced Raman scattering (SERS)," Physical Review Letters, vol. 78, no. 9, pp. 1667-1670, 1997.

[22] X. M. Qian and S. M. Nie, "Single-molecule and singlenanoparticle SERS: from fundamental mechanisms to biomedical applications," Chemical Society Reviews, vol. 37, no. 5, pp. 912-920, 2008.

[23] K. Kneipp, H. Kneipp, I. Itzkan, R. R. Dasari, and M. S. Feld, "Ultrasensitive chemical analysis by Raman spectroscopy," Chemical Reviews, vol. 99, no. 10, pp. 2957-2976, 1999.

[24] T. E. Furtak, G. Trott, and B. H. Loo, "Enhanced light scattering from the metal/solution interface: chemical origins," Surface Science, vol. 101, no. 1-3, pp. 374-380, 1980.

[25] I. Pockrand and A. Otto, "Surface enhanced Raman scattering (SERS): annealing the silver substrate," Solid State Communications, vol. 38, no. 12, pp. 1159-1163, 1981. 
[26] T. Watanabe, N. Yanagihara, K. Honda, B. Pettinger, and L. Moerl, "Effects of underpotentially deposited TI and Pb submonolayers on the surface-enhanced Raman scattering (SERS) from pyridine at Ag electrodes," Chemical Physics Letters, vol. 96, no. 6, pp. 649-655, 1983.

[27] D. P. Tsai, J. Kovacs, Z. H. Wang et al., "Photon scanning tunneling microscopy images of optical excitations of fractal metal colloid clusters," Physical Review Letters, vol. 72, no. 26, pp. 4149-4152, 1994.

[28] P. Zhang, T. L. Haslett, C. Douketis, and M. Moskovits, "Mode localization in self-affine fractal interfaces observed by nearfield microscopy," Physical Review B, vol. 57, no. 24, pp. 15513-15518, 1998.

[29] C. L. Haynes and R. P. Van Duyne, "Nanosphere lithography: a versatile nanofabrication tool for studies of size-dependent nanoparticle optics," Journal of Physical Chemistry B, vol. 105, no. 24, pp. 5599-5611, 2001.

[30] M. J. Weaver, "Surface-enhanced Raman spectroscopy as a versatile in situ probe of chemisorption in catalytic electrochemical and gaseous environments," Journal of Raman Spectroscopy, vol. 33, no. 5, pp. 309-317, 2002.

[31] G. T. Duan, W. P. Cai, Y. Y. Luo, Y. Li, and Y. Lei, "Hierarchical surface rough ordered $\mathrm{Au}$ particle arrays and their surface enhanced Raman scattering," Applied Physics Letters, vol. 89, no. 18, Article ID 181918, 2006.

[32] Z. L. Huang, G. W. Meng, Q. Huang, Y. J. Yang, C. H. Zhu, and C. L. Tang, "Improved SERS performance from Au nanopillar arrays by abridging the pillar tip spacing by Ag sputtering," Advanced Materials, vol. 22, no. 37, pp. 4136-4139, 2010.

[33] M. Malac, R. F. Egerton, M. J. Brett, and B. Dick, "Fabrication of submicrometer regular arrays of pillars and helices," Journal of Vacuum Science and Technology B, vol. 17, no. 6, pp. 26712674, 1999.

[34] B. Dick, M. J. Brett, and T. Smy, "Controlled growth of periodic pillars by glancing angle deposition," Journal of Vacuum Science and Technology B, vol. 21, no. 1, pp. 23-28, 2003.

[35] Q. Zhou, Z. C. Li, Y. Yang, and Z. J. Zhang, "Arrays of aligned, single crystalline silver nanorods for trace amount detection," Journal of Physics D, vol. 41, no. 15, Article ID 152007, 2008.

[36] Q. Zhou, Y. He, J. Abell, Z. Zhang, and Y. Zhao, "Optical properties and surface enhanced raman scattering of L-shaped silver nanorod arrays," Journal of Physical Chemistry C, vol. 115, no. 29, pp. 14131-14140, 2011.

[37] Q. Zhou, Y. J. Liu, Y. P. He, Z. J. Zhang, and Y. P. Zhao, “The effect of underlayer thin films on the surface-enhanced Raman scattering response of Ag nanorod substrates," Applied Physics Letters, vol. 97, no. 12, Article ID 121902, 2010.

[38] Z. Q. Tian, B. Ren, and D. Y. Wu, "Surface-enhanced Raman scattering: from noble to transition metals and from rough surfaces to ordered nanostructures," Journal of Physical Chemistry B, vol. 106, no. 37, pp. 9463-9483, 2002.

[39] X. Zhang, Q. Zhou, J. Ni, Z. C. Li, and Z. J. Zhang, "Surfaceenhanced Raman scattering from a hexagonal lattice of micropatterns of vertically aligned Ag nanorods," Physica E, vol. 44, no. 2, pp. 460-463, 2011.

[40] Y. Z. Chu, M. G. Banaee, and K. B. Crozier, "Double-resonance plasmon substrates for surface-enhanced Raman scattering with enhancement at excitation and stokes frequencies," ACS Nano, vol. 4, no. 5, pp. 2804-2810, 2010.
[41] L. M. Tong, T. Zhu, and Z. F. Liu, "Approaching the electromagnetic mechanism of surface-enhanced Raman scattering: from self-assembled arrays to individual gold nanoparticles," Chemical Society Reviews, vol. 40, no. 3, pp. 1296-1304, 2011.

[42] L. Gunnarsson, E. J. Bjerneld, H. Xu, S. Petronis, B. Kasemo, and M. Käll, "Interparticle coupling effects in nanofabricated substrates for surface-enhanced Raman scattering," Applied Physics Letters, vol. 78, no. 6, pp. 802-804, 2001.

[43] L. A. Dick, A. J. Haes, and R. P. Van Duyne, "Distance and orientation dependence of heterogeneous electron transfer: a surface-enhanced resonance Raman scattering study of cytochrome c bound to carboxylic acid terminated alkanethiols adsorbed on silver electrodes," Journal of Physical Chemistry B, vol. 104, no. 49, pp. 11752-11762, 2000.

[44] L. A. Dick, A. D. McFarland, C. L. Haynes, and R. P. Van Duyne, "Metal film over nanosphere (MFON) electrodes for surface-enhanced Raman spectroscopy (SERS): improvements in surface nanostructure stability and suppression of irreversible loss," Journal of Physical Chemistry B, vol. 106, no. 4, pp. 853-860, 2002.

[45] M. Litorja, C. L. Haynes, A. J. Haes, T. R. Jensen, and R. P. Van Duyne, "Surface-enhanced Raman scattering detected temperature programmed desorption: optical properties, nanostructure, and stability of silver film over $\mathrm{SiO}_{2}$ nanosphere surfaces," Journal of Physical Chemistry B, vol. 105, no. 29, pp. 69076915, 2001.

[46] J. A. Dieringer, A. D. McFarland, N. C. Shah et al., "Surface enhanced Raman spectroscopy: new materials, concepts, characterization tools, and applications," Faraday Discussions, vol. 132, pp. 9-26, 2006.

[47] C. R. Yonzon, C. L. Haynes, X. Y. Zhang, J. T. Walsh, and R. P. Van Duyne, "A glucose biosensor based on surface-enhanced Raman scattering: improved partition layer, temporal stability, reversibility, and resistance to serum protein interference," Analytical Chemistry, vol. 76, no. 1, pp. 78-85, 2004.

[48] A. Tao, F. Kim, C. Hess et al., "Langmuir-Blodgett silver nanowire monolayers for molecular sensing using surfaceenhanced Raman spectroscopy," Nano Letters, vol. 3, no. 9, pp. 1229-1233, 2003.

[49] G. Ross, "The public health implications of polychlorinated biphenyls (PCBs) in the environment," Ecotoxicology and Environmental Safety, vol. 59, no. 3, pp. 275-291, 2004.

[50] Q. Zhou, Y. Yang, J. Ni, Z. C. Li, and Z. J. Zhang, "Rapid detection of 2, 3, 3' $, 4,4^{\prime}$-pentachlorinated biphenyls by silver nanorods-enhanced Raman spectroscopy," Physica E, vol. 42, no. 5, pp. 1717-1720, 2010.

[51] Q. Zhou, X. Zhang, Y. Huang, Z. C. Li, and Z. J. Zhang, "Rapid detection of polychlorinated biphenyls at trace levels in real environmental samples by surface-enhanced Raman scattering," Sensors, vol. 11, no. 11, pp. 10851-10858, 2011.

[52] Q. Zhou, Y. Yang, J. E. Ni, Z. C. Li, and Z. J. Zhang, "Rapid recognition of isomers of monochlorobiphenyls at trace levels by surface-enhanced Raman scattering using Ag nanorods as a substrate," Nano Research, vol. 3, no. 6, pp. 423-428, 2010.

[53] X. Zhang, Q. Zhou, Y. Huang, Z. C. Li, and Z. J. Zhang, "Contrastive analysis of the Raman spectra of polychlorinated benzene: hexachlorobenzene and benzene," Sensors, vol. 11, no. 12, pp. 11510-11515, 2011.

[54] Y. Cao, R. C. Jin, and C. A. Mirkin, "Nanoparticles with Raman spectroscopic fingerprints for DNA and RNA detection," Science, vol. 297, no. 5586, pp. 1536-1540, 2002. 


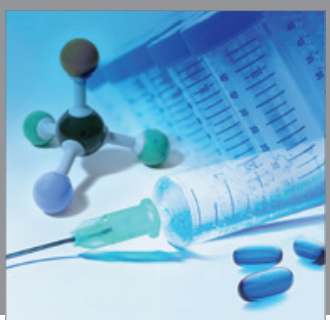

International Journal of

Medicinal Chemistry

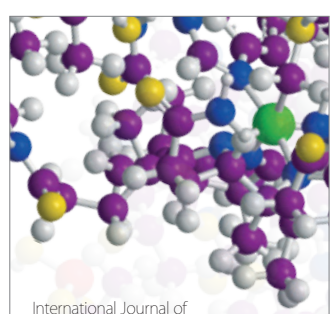

Carbohydrate Chemistry

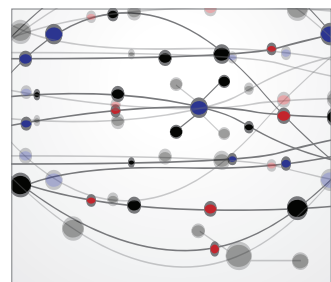

The Scientific World Journal
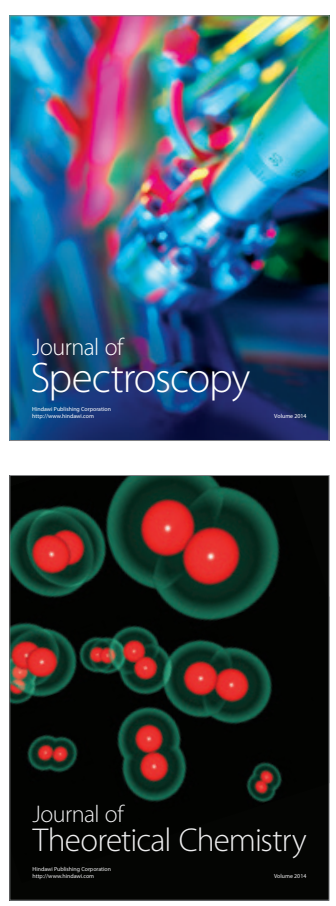
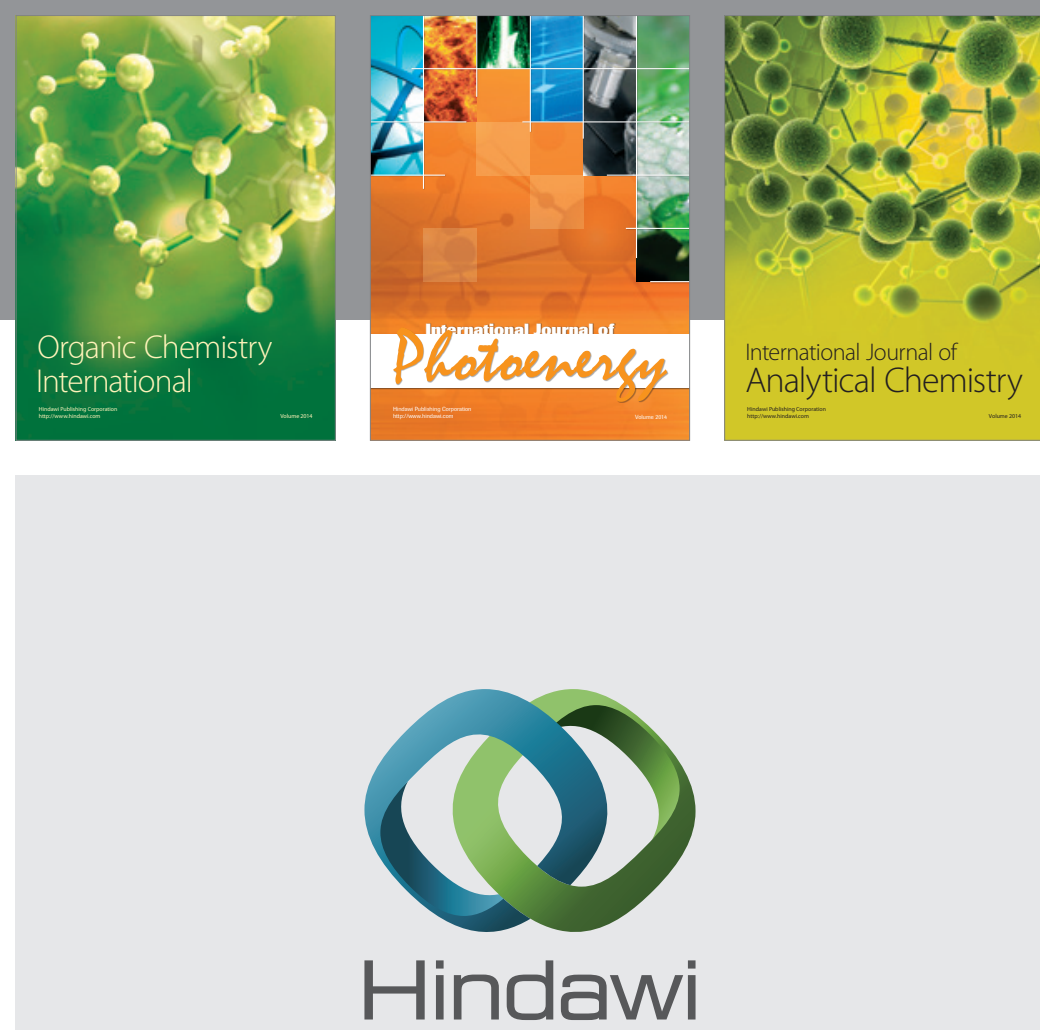

Submit your manuscripts at

http://www.hindawi.com
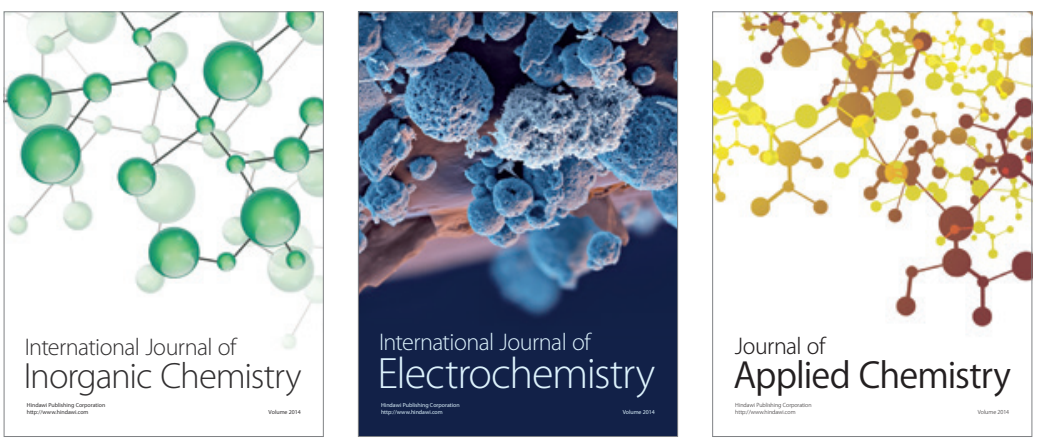

Journal of

Applied Chemistry
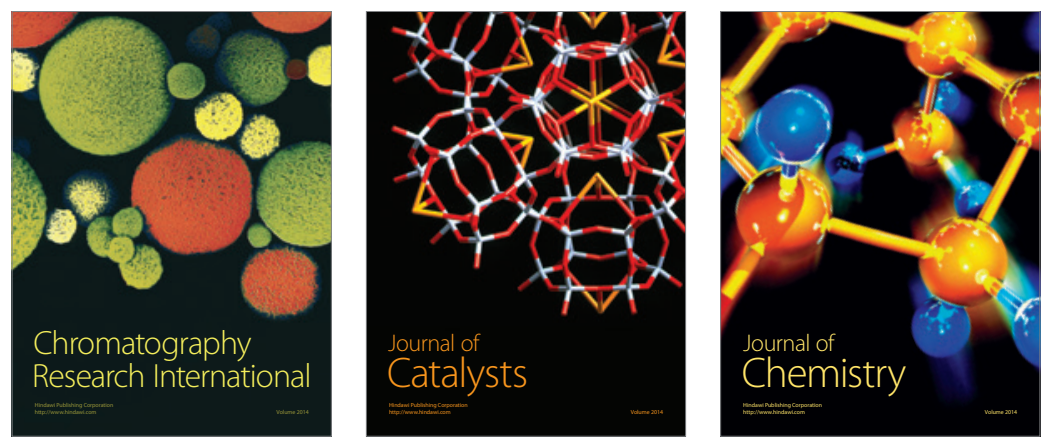
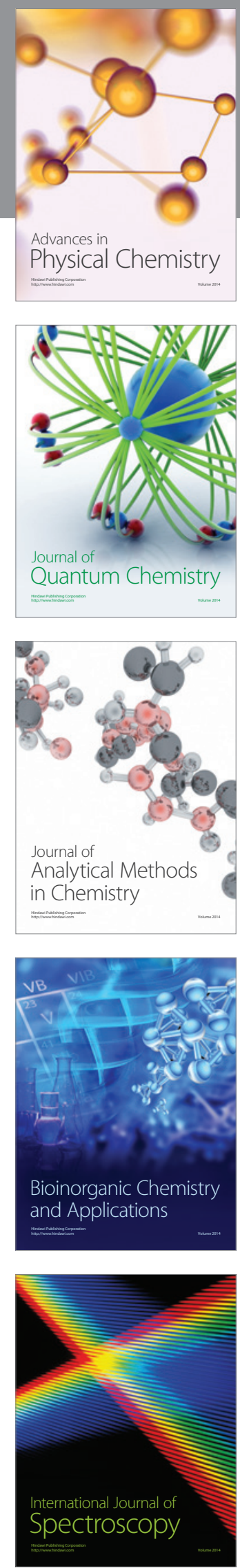